\title{
Dinzelbacher, Peter, Heinz, Werner, Europa in der Spätantike 300-600. Eine Kultur- und Mentalitätsgeschichte
}

\section{Thomas Lienhard}

\section{(2) OpenEdition \\ Journals}

Édition électronique

URL : http://journals.openedition.org/ifha/1637

DOI : 10.4000/ifha.1637

ISSN : 2198-8943

Éditeur

IFRA - Institut franco-allemand (sciences historiques et sociales)

Référence électronique

Thomas Lienhard, « Dinzelbacher, Peter, Heinz, Werner, Europa in der Spätantike 300-600. Eine Kulturund Mentalitätsgeschichte », Revue de l'IFHA [En ligne], Date de recension, mis en ligne le 01 janvier 2008, consulté le 22 septembre 2020. URL : http://journals.openedition.org/ifha/1637 ; DOI : https:// doi.org/10.4000/ifha.1637

Ce document a été généré automatiquement le 22 septembre 2020.

(CIFHA 


\title{
Dinzelbacher, Peter, Heinz, Werner, Europa in der Spätantike 300-600. Eine Kultur- und Mentalitätsgeschichte
}

\author{
Thomas Lienhard
}

1 Ce livre n'a pas l'ambition de présenter des recherches originales ; il n'est pas non plus un manuel ; et pourtant il rendra bien des services à la communauté universitaire.

2 Comme souvent, les indications essentielles concernant le contenu de l'ouvrage se trouvent dans le sous-titre. Celui-ci dissuadera le lecteur de chercher des renseignements détaillés à propos des évolutions politiques (de fait, elles occupent moins de cinq pages), économiques (une demi-page) ou de l'histoire de l'Église. Que reste-t-il ? Plutôt que de rappeler ici les problèmes de définition de l'histoire des mentalités, ou de traduction de la Kulturgeschichte, contentons-nous de présenter les thèmes effectivement abordés. Dans une première partie intitulée « la perception de soi ", les auteurs présentent les conceptions du bonheur et de l'esthétique (notamment corporelle), les phénomènes religieux dans un sens très large, le rapport à la maladie, à la mort et à la peur, puis les modes de raisonnement. Dans une deuxième partie consacrée aux relations sociales, ils évoquent les différents cercles sociaux de manière concentrique (le couple et la famille, y compris dans leurs aspects érotiques et éducatifs, les amis, les réseaux hiérarchiques et le rapport à l'État) ainsi que l'évolution $\mathrm{du}$ droit et des moyens de communication. Une troisième partie analyse les relations entre l'humain et son environnement naturel : au lieu de considérer celui-ci comme un fondement de la vie sociale comme l'aurait fait tout ouvrage historique il y a un demisiècle (vraisemblablement en plaçant ce chapitre en tête de l'ouvrage), les auteurs estiment qu'il ne s'agissait là que d'un aspect parmi d'autres de la vie mentale, impliquant notamment la préhension scientifique du monde naturel et le rapport à l'espace ou au temps. On est ainsi à mi-chemin entre l'histoire de la vie quotidienne, de la culture et des mentalités, et bien près des nouveaux objets d'histoire naguère appelés par Jacques Le Goff et Pierre Nora, mais rarement étudiés pour cette période. Si l'on ajoute à cette originalité du propos la splendeur des illustrations proposées, en 
particulier des reconstitutions architecturales, on admettra sans peine que pour l'enseignant comme pour celui qui souhaite découvrir cette période, cet ouvrage fournira un outil précieux ; on peut d'ailleurs signaler qu'il ne constitue qu'un chaînon dans une série qui couvre déjà l'ensemble du Moyen Âge (voir notamment BullMHFA, 43, 2007, p. 302-303).

Comme prix de cette synthèse originale, le lecteur historien devra parfois concéder une livre de sa chair et consentir à des schémas exagérément simplificateurs. Tel est le cas notamment des présentations récurrentes à propos des Germains, des Romains et des chrétiens, les premiers étant campés dans le rôle suranné d'un groupe plus mobile socialement et plus favorable à la liberté des femmes, les seconds comme une société sexuellement libérée à laquelle les troisièmes auraient apporté un rigorisme et un refus du monde inédits... Ces archaïsmes historiographiques proviennent peut-être en partie de la difficulté éprouvée par les deux auteurs (ils ne sont pas les seuls !) à trancher entre les divers modèles actuellement invoqués pour expliquer les évolutions sociales de l'Antiquité tardive, et notamment du rôle que jouèrent les Barbares dans ce processus : ainsi, alors que P.D. et W.H. reprennent un schéma très classique dans leur introduction (les Huns auraient chassé les Goths qui auraient chassé les autres peuples germaniques qui auraient finalement envahi et transformé le monde romain), ils remettent eux-mêmes cette présentation en cause en conclusion, rappelant alors les modèles plus récents, plus nuancés et plus justes de l'ethnogénèse. La simplification glisse ici vers la contradiction interne.

4 L'ouvrage reste pourtant fort utile parce qu'il dispose d'un quasi-monopole à propos des thématiques étudiées, en particulier dans le domaine francophone où l'Antiquité tardive, a fortiori étudiée sous l'angle des mentalités, est un champ en jachère. Une petite analyse de la bibliographie fournie par les auteurs est d'ailleurs significative : sur les quelque 500 titres référencés, moins de $5 \%$ ont été produits par des historiens français, et encore un tiers d'entre eux date-t-il d'avant 1950. Dans ce contexte, l'ouvrage de P.D. et de W.H. n'a pas besoin d'être une recherche originale ni un manuel exhaustif : il est un livre utile, en même temps qu'un plaisir. 